\title{
Embolization of Atrial Septal Defect Occluder Device into the Left Ventricle- Emergency Surgical Removal.
}

\author{
SANJAY KUMAR RAHA, SWADESH RANJAN SARKER , ATS AHMED , MD. ZAHIDUL ISLAM, \\ MD. REZAUL KARIM ,MD. KAMRUL HASAN \\ Department of Cardiac Surgery, National Institute of Cardiovascular Diseases (NICVD), Dhaka
}

Address for Correspondance: Professor Md. Kamrul Hasan, Professor and Head, Department of Cardiac Surgery

NICVD, Dhaka.E-mail-kamrul.dmc44@yahoo.com

\begin{abstract}
:
With the introduction of technological improvements, invasive cardiologic interventions have become increasingly used alternatives to surgery. Despite its numerous benefits, serious and potentially life-threatening complications of invasive cardiology interventions may occur. We presented a case of attempted percutaneous transcatheter closure of a large ASD in 6 years old boy, complicated by device embolization to the left ventricle necessitating emergency surgical retrieval in the department of cardiac surgery, National Institute of Cardiovascular Diseases (NICVD), Dhaka.
\end{abstract}

Keywords: Atrial septal defect (ASD), occluder devices, Embolization.

\section{Introduction:}

Atrial septal defect (ASD) is the prevalent congenital heart disease (3.78 per 10000 live births). ${ }^{1}$ For several decades surgical closure has been considered the standard method of repairing a secundum ASD. Surgical treatment is safe and effective but the complications relate to thoracotomy, bleeding, arrhythmia, post-pericardiectomy syndrome, and residual defects. ${ }^{1}$ There have been some creative efforts by interventional cardiologists in closing ASD using various devices. ASD closure device was first described by Ring et al. in 1976. ${ }^{1}$ Percutaneous ASD closure has become an increasingly simplified procedure over the past decade. Complications commonly associated with ASD closure device included residual shunt, device malposition or embolization, thrombosis over the vena cava or atrium, erosion and perforation of the heart, and infective endocarditis. ${ }^{2}$ Device embolization or erosion could result in catastrophic complications such as left ventricle outflow tract obstruction, cardiac rupture or cardiac tamponade. Chun reported a case with aorta-to-right atrial fistula 3 months after device implantation. ${ }^{2}$ The use of dedicated devices for ASD closure by experienced operators results in low rates of device dislodgement, migration or embolization and improves sealing performance. A cause of failure is early embolization. The percentage of this early embolization is around $0.5 \%$. These have resulted in varying symptom and hemodynamic compromise, most requiring surgical retrieval. ${ }^{3}$ We report a case of ASD closure device embolization to left ventricular inflow.

\section{Case report:}

A 6 year old boy with symptoms of pal-pitation and fatigue underwent transthoracic echocardiography, which showed a $15 \mathrm{~mm}$ secundum ASD having well defined rim with left to right shunt and LVEF of $77 \%$. The patient was therefore seemed to be a good candidate for ASD closure using $16 \mathrm{~mm}$ Amplatzer Septal Occluder (ASO). Standard practice was adopted for deployment of the device. After the device was released, it slipped entirely into the left atrium, protruding into the mitral orifice and then to left ventricle. Retrieval efforts were fraught with the risk of mitral disruption or occlusion and were eventually unsuccessful. Although the patient was haemodynamically stable, urgent surgical intervention was recommended to retrieve the device and close the defect. The patient was taken emergently to the operating theatre, median sternotomy was done and cardiopulmonary bypass was established by aortic and bicaval cannulation. The aorta was clamped and heart was arrested with antegrade cardioplegia. The right atrium was opened and a large secundum ASD was found. The device was covered with recent red thrombus and was found to be embolized entirely into the left ventricle, lying just below the mitral valve. Through the ASD, the device was retrieved without injury to the mitral valve and the defect was closed with gluteraldehyde treated autologous pericardial patch. Patient was gradually weaned from cardiopulmonary bypass. Patient was extubated after 4 hours of mechanical ventilation. The patient had an uneventful recovery and was discharged a week later. 


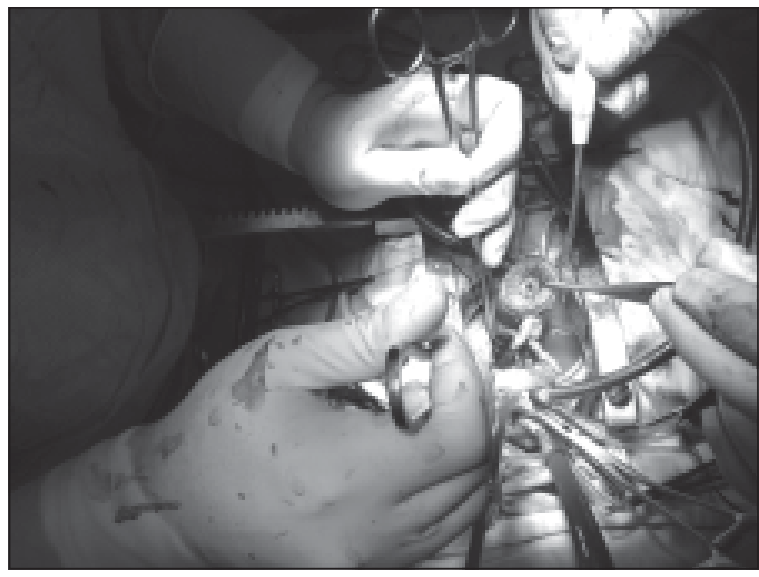

Fig.-1: Surgical Retrieval of ASD Device

\section{Discussion:}

Transcatheter occlusion techniques have become an increasingly used alternative to surgical closure in selected cases of the ASD and various devices are available for this. ${ }^{3}$ Most ASD secundums can be closed by Amplatzer device. Common criteria for a patient being suitable for closure by Amplatzer include a defect with less than or equal to $34 \mathrm{~mm}$, a $4 \mathrm{~mm}$ rim of septal tissue surrounding the defect and sufficient distances from surrounding valves and structures. The hazards of ASO are thick profile of device and high amount of nitinol in device and the potential for nikle toxicity. ${ }^{1}$ Transcatheter closure of ASD has comparable results to surgical closure ${ }^{3}$. Absence of residual shunts and late thromboembolic events is in favor of surgical closure of ASD. Minimally invasive techniques address cosmetic angle without compromising results. The need for lifelong antiplatelet agents and SBE prophylaxis has to be weighed against the disadvantage of a small incision. A promising early result by device closure does not guarantee a favorable late outcome. ${ }^{4}$ Embolization does not commonly occur but if develops, the reasons are insufficient rim around the defect, early release of device, and mismatch between the size of ASD and Amplatzer which is due to the use of small Amplatzer for a large ASD. Transesophageal echocardiography (TEE) performed during Amplatzer placement seems to be a potential contributor to prevent technical errors and occurrence of complications. ${ }^{1}$ Transcatheter devices can be associated with failures and most importantly, with lifethreatening complications. The most important reason for acute failure of these devices is poor patient selection, device selection, or both. ${ }^{5}$ Other suggested mechanisms of acute failure are device related failure, inadequate experience, poor defect rim to hold the device, and tearing of the interatrial septum as a result of catheter and device manipulations. A part or the entire device might embolize to the right or left atrium, main pulmonary artery, or even to other parts of the vascular tree on both the right and left sides of the circulation. Once the device is detached from its cable, it becomes difficult to retrieve, and depending on its location, it might even become lethal. ${ }^{5}$ Hui Chung Wu et al. (2013) mentioned in their study that between January 2000 and December 2010, a total of 508 patients underwent closure of secundum type ASD using an Amplatzer septal occluder (ASO). Six of the patients (1.2\%) had device embolization or migration warranting surgical retrieval and repair. ${ }^{2}$ In a report by David et al. 186 patients had atrial defects closed by means of Amplatzer device with no major complications. There were 8 (4.3\%) minor complications including 4 device embolization with 2 removed percutaneously and 2 surgically. Also, 4 instances of arrhythmia comprising 3 transient, and 1 persistent complete heart block. ${ }^{1}$ Percutaneous retrieval of the embolized device is possible in about $70 \%$ of cases. However, some authors suggest that embolization of device is always an indication for emergency surgical retrieval, which also permits a direct inspection of intra-cardiac structures that may have become injured. ${ }^{6}$ In our patient, we also preferred surgical approach due to close relation of device with mitral valve in the left ventricle. In conclusion, a careful echocardiographic assessment and procedure planning should be done for a percutaneous intervention. In addition, surgical back-up must be available in the hospital to cope with potentially lethal acute complications.

Although closure of left to right shunting defects by percutaneous occluder devices has a lot of advantages, device embolization is still a major complication. Surgical management is the only method to remove embolized devices after failed percutaneous retrieval attempts. In this circumstance, surgical back-up and urgent management strategies should be well planned.

\section{References:}

1. Tabaee AS, Rostami AR, Ghafari R. Surgical removal of an embolized amplatzer device from left ventricle. Iranian Cardiovascular Research Journal 2007; 1:53-57.

2. Wu HC, Wang CC, Fu YC. Surgical Management for Complications during Closure of Atrial Septal Defect with Amplatzer Device. Acta Cardiol Sin 2013; 29:557-61.

3. Yetkin U, Yurekli I, Akyildiz ZI. Emergency surgical approach to device emboli due to migration of the atrial septal defect occlude. Arch Med Sci 2014; 10: 464-69.

4. Raghuram AR, Krishnan R, Kumar S. Complications in atrial septal defect device closure. Interactive CardioVascular and Thoracic Surgery 2008; 7:167-69.

5. Contrafouris C A, Chatzis AC, Giannopoulos NM. Emergency Surgery for Embolization of Amplatzer device into the Left Atrium. Hellenic J Cardiol 2006; 47: 245-46.

6. Seren M, Çiçek OF, Düzgün AC. Surgical treatment of two different interventional cardiological complications at the same patient. Cardiovascular Surgery and Interventions 2014;1:6-8. 